\title{
Flu soothes with stress hormones
}

\begin{abstract}
Although much is known about immunity to single viral and bacterial pathogens, much less is known about how an ongoing immune response to one pathogen affects immunity to another. This is a clinically important issue as fatalities following influenza virus infection are often associated with secondary bacterial infections. In this study, Jamieson et al. show that influenza virus infection suppresses immunity to systemic Listeria monocytogenes infection through the induction of glucocorticoids but that virusinduced glucocorticoids are required to prevent lethal immunopathology in co-infected mice.

To understand the effect of influenza virus infection on the systemic immune response to bacteria, mice were infected intranasally with influenza virus and subsequently infected intravenously with $L$. monocytogenes. Mice that were infected with both pathogens had increased bacterial
\end{abstract}

burden in the liver compared with mice infected with $L$. monocytogenes only. This increase in bacterial burden was accompanied by a decrease in immune cell infiltration and decreased expression of chemokines, such as CC-chemokine ligand 7 (CCL7), and intercellular adhesion molecule 1 (ICAM1) in the liver. Serum levels of interleukin-6 (IL-6) and interferon- $\gamma$ (IFN $\gamma$ ) and total splenocyte numbers were also decreased in co-infected mice. The increase in bacterial burden in the liver was not due to virus-induced type I IFN production, which is known to decrease resistance to L. monocytogenes infection, and was also independent of the adaptive immune system.

So, how does influenza virus infection suppress systemic immune responses to secondary bacterial infections? Increased and sustained levels of glucocorticoids were detected in the serum of virusinfected or co-infected mice, but

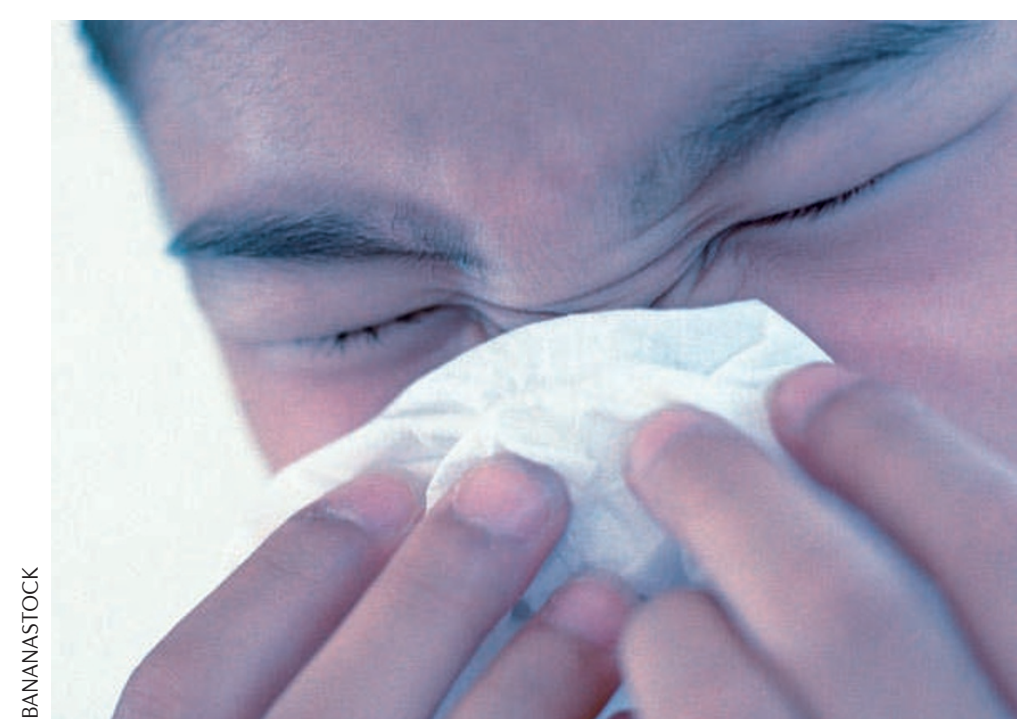

only a transient increase in serum glucocorticoid levels was observed in mice infected with just bacteria. The virus-induced increase in glucocorticoids was shown to be independent of a systemic inflammatory response. Co-infection of adrenalectomized (ADX) mice, which cannot produce glucocorticoids, resulted in bacterial burden in the liver similar to that in mice infected with L. monocytogenes alone. Furthermore, the levels of cell infiltration and CCL7 and ICAM1 expression in the liver, and of IL-6 in the blood, of co-infected ADX mice were similar to mice infected with L. monocytogenes alone. These data indicate that the loss of glucocorticoid production prevents virus-induced immunosuppression of systemic bacterial responses.

However, ADX but not wild-type mice succumbed to co-infection with sub-lethal doses of the two pathogens. This was accompanied by a large increase in serum levels of tumour necrosis factor and the p40 subunit of IL-12, as well as increased immunopathology in the lungs but not the liver.

So, influenza virus infection induces high and sustained levels of glucocorticoids, probably through virus-induced lung-tissue damage, which suppress systemic immune responses to secondary bacterial infections but are needed to protect co-infected mice from lethal immunopathology.

Olive Leavy

ORIGINAL RESEARCH PAPER Jamieson, A. M., Yu, S., Annicelli, C. H. \& Medzhitov, R. Influenza virus-induced glucocorticoids compromise innate host defense against a secondary bacterial infection. Cell Host Microbe 7, 103-114 (2010) 